\title{
Study on Preparation and Characterization of Graphene Based on Ball Milling Method
}

\author{
Gang Huang, ${ }^{1}$ Chuncheng Lv $\mathbb{D}^{1,2}$ Junxi He, ${ }^{1}$ Xia Zhang, ${ }^{1}$ Chao Zhou, ${ }^{1}$ Pan Yang, ${ }^{1}$ Yan Tan, \\ and Hao Huang ${ }^{1}$ \\ ${ }^{1}$ National and Local Joint Engineering Laboratory of Traffic Civil Engineering Materials, Chongqing Jiaotong University, \\ Chongqing 400074, China \\ ${ }^{2}$ Dazhou Urban and Rural Planning Development Center, Sichuan 635000, China
}

Correspondence should be addressed to Chuncheng Lv; 622170951108@mails.cqjtu.edu.cn

Received 6 April 2020; Revised 19 November 2020; Accepted 27 November 2020; Published 12 December 2020

Academic Editor: Raul Arenal

Copyright ( $) 2020$ Gang Huang et al. This is an open access article distributed under the Creative Commons Attribution License, which permits unrestricted use, distribution, and reproduction in any medium, provided the original work is properly cited.

\begin{abstract}
Taking advantage of the state-of-the-art graphene preparation technologies in China and abroad, this work studied the preparation processes of graphene for road applications based on the preliminary high-speed vibration ball milling method. This work combined numerical modeling and microscopic experiments. The preparation parameters were optimized through a multifactor and multilevel test scheme. The materials, influencing factors, and parameters in the preparation process were systematically studied. A graphene preparation method was proposed that considered vibration frequency, static filling rate, material-to-ball volume ratio, and the void percentages of media. Flake graphite, aluminum powder, and 304 stainless-steel grit were used as the preparation materials. The preparation parameters and process model were established based on the uniform design method. The preparation parameters were proposed, calculated, and optimized. Microscopic analysis showed that the proposed preparation method can improve the quality of graphene. This study provides a new source of raw materials for the application of graphene in road engineering.
\end{abstract}

\section{Introduction}

Graphene has attracted widespread attention from researchers in China and abroad since the discovery of fullerene, a quasizero-dimensional material, in the 1980s. In 2004, two British scientists (Andre K. Geim and Kanstantin Novoselov) successfully isolated graphene, a two-dimensional carbon nanomaterial composed of single-layer carbon atoms, by peeling pyrolytic graphite sheet with adhesive tape [1-3]. Graphene is a two-dimensional honeycomb-like nanomaterial made of $\mathrm{sp}^{2}$-hybridized carbon atoms. The strong intermolecular force $(\pi-\pi$ interaction) between graphene molecules makes the graphene structure very stable [4]. Graphene has high transmittance (97.7\%) [5], high Young's modulus (1 TPa) $[6,7]$, high specific surface area $\left(2630 \mathrm{~m}^{2} / \mathrm{g}\right)[8]$, high thermal conductivity $(5000 \mathrm{~W} / \mathrm{m} \cdot \mathrm{K})$, and high carrier mobility $\left(2.5 \times 10^{5} \mathrm{~cm}^{2} /(\mathrm{V} \cdot \mathrm{s})\right)$ [9]. It has received widespread attention in the fields of biology, medicine, environmental science, electronics, energy, and materials science because of its unique physical structure and excellent electrical, optical, thermal, and mechanical properties [10].

It is particularly difficult to peel off graphene nanosheets from graphite because of the van der Waals force between the graphite layers. In the past decade, graphene preparation technology has developed rapidly in China and abroad with the widespread attention given to carbon nanomaterials. At present, common graphene preparation methods include mechanical exfoliation, oxidation-reduction, liquid-phase exfoliation, electrochemical exfoliation, supercritical fluid exfoliation, chemical vapor deposition (CVD), and epitaxial growth [11-16]. Graphenes that are prepared by different methods have different qualities and application prospects. For example, CVD-prepared graphene sheets have a large area, good uniformity, high quality, and controllable number of layers. They are suitable for high-end fields such as optics, electronics, sensors, energy, and biology. However, they are 
rarely used as road materials because of the strict preparation process and high cost. Currently, graphene oxide and (nonfunctional group) graphene are used in the road field. Relevant research at home and abroad mainly focuses on the performance of graphene composite road materials, For example, Habib et al.'s [17] research suggests that graphene oxide asphalt helps improve the resistance to deformation of the road surface and reduce rutting in the road surface. According to Li's [18] research, the high-temperature performance and plasticity of the graphene-modified asphalt have been significantly improved, He's [19] research shows that the addition of graphene improves the low temperature stability of asphalt to a certain extent. However, there is no research on the preparation process of road graphene. The mechanical exfoliation method is relatively simple. Graphene materials prepared by this method have low size requirements and low cost. High-performance graphene composite road materials can be developed by combining the mechanically exfoliated graphene with road engineering materials to employ the advantages of the graphene. This study was funded by the National Natural Science Foundation of China. According to a patented graphene preparation technology, this study aimed to develop graphene with good quality and low cost based on mechanical exfoliation and ball milling. A numerical method was employed to optimize the graphene preparation parameters. This work provides a new source of raw materials for the application of graphene in road engineering and provides a reference for the development of graphene composite road materials [20-27].

\section{Preparation of Graphene by Ball Milling}

2.1. Materials and Equipment. In this study, a QM-3B high-speed vibrating ball mill (specification: $80 \mathrm{ml}$, vibration frequency: $1200 \mathrm{r} / \mathrm{min}$, motor specification: $220 \mathrm{~V}$, $180 \mathrm{~W}$ ) produced by Nanjing University Instrument Factory was used. X-ray diffraction (XRD) was performed with a D8ADVANCE (Bruker, Germany) X-ray diffractometer (Cu$\mathrm{K} \alpha$ radiation source, voltage $40 \mathrm{kV}$, current $40 \mathrm{~mA}$, scan speed $0.1 \mathrm{sec} / \mathrm{step}$, wavelength $1.5418 \AA$ ). The specific surface area was measured on a Quadrasorb SI analyzer (Quantachrome Instruments). High-resolution transmission electron microscopy (HRTEM) was performed with a Tecnai G2 F20 field emission transmission electron microscope (FEI, USA).

The materials used in this study included aluminum (Tenghui Metal Material Co., Ltd., Qinghe, China; particle size: $1.2 \mathrm{~mm}, 40$ mesh, 80 mesh, 100 mesh, 200 mesh, and 300 mesh; AR $\geq 99.00 \%$ ), 304 stainless-steel grit (Lianzhiyan Surface Treatment Material Co., Ltd., Dongguan, China; particle size: $0.2 \mathrm{~mm}, 0.4 \mathrm{~mm}$, and $0.8 \mathrm{~mm}$ ), and flake graphite (Qingdao Chenyang Graphite Co., Ltd., Qingdao, China; particle size: 50 mesh).

2.2. Working Principle of Ball Milling. In this study, graphene was prepared using a QM-3B high-speed vibrating ball mill (specification: $80 \mathrm{ml}$; vibration frequency: $1200 \mathrm{r} / \mathrm{min}$; motor specification: $220 \mathrm{~V}, 180 \mathrm{~W}$ ) [28]. 304 stainless-steel balls were used as grinding balls. The working principle is as follows: the high-speed rotation of the motor drives the cylinder to rotate at a high speed on the springs. This process is accompanied by simple harmonic vibration. During the rotation process, the frictional resistance between the cylinder and the steel ball causes accelerated motion of the steel ball. The kinetic energy gained by the steel ball is then transferred to the material as the ball collides with the material and other steel balls. As a result, the material is ground by the balls. At the same time, the generated vibration prevents the steel balls from centrifugal movement due to the high-speed rotation and provides more impact energy to ensure the balls have enough kinetic energy. Therefore, the kinetic energy of the high-speed vibrating ball mill is generally greater than that of the planetary ball mill. However, the cylinder volume of the high-speed vibrating ball mill is only $80 \mathrm{ml}$. Its grinding capacity is small, so this type of ball mill is mostly used for laboratory grinding studies. There are few studies on the working parameters of high-speed vibrating ball mills. The parameters are generally designed based on the theoretical calculation of tumbling ball mills and are optimized based on experience $[29,30]$. González-Domínguez [31] prepared graphene by planetary ball milling. The raw material ratio is only $25 \mathrm{mg}$, and the ball mill speed is only $100 \mathrm{r} / \mathrm{min}$. In Buzaglo's [32] study, the content of graphite was only $36 \mathrm{mg}$. In summary, these two ball milling methods have low production efficiency and high cost, so they are not suitable for road materials.

2.3. Influencing Factors of Grinding Efficiency. During the ball milling process, the rotation and vibration of the cylinder drive the steel balls and materials to rise. Then, the steel balls and materials drop into the cylinder. The steel balls and the materials crush and collide with each other to generate impact energy, shear energy, and friction energy, thereby achieving the purpose of grinding. The grinding process of the ball mill is essentially the movement process of discrete particles. At present, most of the domestic and foreign studies on the grinding process of ball mills determine the relevant parameters by discrete element methods and molecular dynamics [33-35]. The ball mill grinding is a complex process with many influencing factors, and these influencing factors restrict and affect each other. The grinding efficiency of the ball mill is determined by the rotation speed of the ball mill, the grinding media (material, size, static filling rate, etc.), the material-to-ball volume ratio (MBVR), the grading of the grinding media, and the grinding time.

(1) The rotation speed is defined as the ratio of the actual rotation speed $n$ to the critical rotation speed $n_{C}$, which is denoted as $\Psi$ :

$$
\Psi=\frac{n}{n_{c}} \times 100 \%
$$

(2) The static filling rate refers to the ratio of the loose volume of the material to the effective volume of the ball mill in the static state, which is denoted $\varphi$, where $R$ is the radius of the cylinder and $h$ is the distance 
TABLE 1: High-speed vibrating ball mill parameters.

\begin{tabular}{|c|c|c|c|c|c|c|c|}
\hline Model & $\begin{array}{c}\text { Cylinder } \\
\text { specification }\end{array}$ & $\begin{array}{c}\text { Motor } \\
\text { specification }\end{array}$ & $\begin{array}{l}\text { Vibration } \\
\text { frequency }\end{array}$ & $\begin{array}{l}\text { Grinding } \\
\text { media }\end{array}$ & $\begin{array}{l}\text { Grinding medium } \\
\text { grading }\end{array}$ & Filling rate & $\begin{array}{l}\text { Grinding medium } \\
\text { void percentage, } \mu\end{array}$ \\
\hline & & & & & $8 \mathrm{~mm} 10 \mathrm{~g}$ & & \\
\hline \multirow[t]{2}{*}{ QM-3B } & $80 \mathrm{ml}$ & $220 \mathrm{~V} 180 \mathrm{~W}$ & $1200 \mathrm{r} / \mathrm{min}$ & 304 stainless-steel grit & $5 \mathrm{~mm} 70 \mathrm{~g}$ & $40 \%$ & 0.38 \\
\hline & & & & & $3 \mathrm{~mm} 98 \mathrm{~g}$ & & \\
\hline
\end{tabular}

from the center of the cylinder bottom to the top surface of the material

$$
\varphi=\frac{1}{\pi}\left\{\arccos \left(\frac{h}{R}\right)-\left(\frac{h}{R}\right)^{2} \operatorname{tg}\left[\arccos \left(\frac{h}{R}\right)\right]\right\} .
$$

(3) The MBVR is the ratio of the volume of the material $V_{m}$ to the void volume between the steel balls $V_{t}$ in the static state, which is denoted $\varphi_{\mathrm{m}}$

$$
\varphi_{m}=\frac{V_{m}}{V_{t}} \times 100 \%
$$

(4) The void percentage of the media is defined as the ratio of the void volume to the bulk volume of the grinding media, which is denoted $\mu . V_{\text {media }}$ is the total volume of the media including voids, $V_{\text {single }}$ is the volume of a single grinding medium particle, and $N$ is the number of medium particles

$$
\mu=\frac{V_{\text {media }}-N V_{\text {single }}}{V_{\text {media }}} \text {. }
$$

In studying the grinding process, there are many differences in the boundary conditions between the theoretical calculation and the actual process. For example, the lubricating effect of the graphite will reduce the frictional resistance between the cylinder and the steel balls; relative sliding movement exists between the steel balls and the cylinder, which lowers the proportion of useful work and the mechanical efficiency; the grading of the steel balls will change the way energy is transferred. It is difficult to apply the theoretical calculation results directly to the actual process. Therefore, the process parameters need to be determined by a combination of theoretical calculation and empirical analysis. Peng Huang, Guojun Shi, and others have performed discrete element simulation analysis on ball mills of different specifications and found that the optimal rotation speed was $80-85 \%$. According to production practices, the grinding effect is good when the filling rate is in the range of $20-50 \%$, and the percentage of voids between the steel balls is 0.35 [36-47].

2.4. Process Parameters of Graphene Preparation by a Ball Mill. The raw material used to prepare graphene was flake graphite [48]. In the study of Prato and Regev [31, 32], melamine and fully conjugated aromatic diluents were used as grinding aids, respectively. However, the surface energy and cohesive energy of molecular crystals are much smaller than that of aluminum; the aluminum powder is used as the grinding aid in this experiment. Graphite is a crystalline mineral of pure carbon. The crystal lattice of graphite has a hexagonal layered structure, and the layered structures can slide against each other in parallel direction. Aluminum has strong plasticity and ductility. The surface of unoxidized aluminum has a strong adsorption capacity. During the ball milling process, the kinetic energy and shear energy generated by the high-speed impact of the grinding balls can break the aluminum to form fresh aluminum surfaces. The surface of the graphite is continuously adsorbed by the freshly generated aluminum surface. The impact from the balls causes the fresh aluminum to peel off the graphite layer by layer, yielding a layered structure of graphite with graphene in the product.

According to the theoretical calculation, the filling rate was kept between $40 \%$ and 50\%. In Prato's study, ten stainless steel balls ( $1 \mathrm{~cm}$ in diameter) were used for grinding, but one size of steel balls would make the energy transfer efficiency lower, so three grades of steel balls were used $(8 \mathrm{~mm}$ particle size, $10 \mathrm{~g}, 5$ pieces; $5 \mathrm{~mm}$ particle size, $70 \mathrm{~g}, 137$ pieces; $3 \mathrm{~mm}$ particle size, $98 \mathrm{~g}$, 889 pieces). As the rotation speed of the high-speed vibrating ball mill was not adjustable, the factory default setting of $1200 \mathrm{r} / \mathrm{min}$ was used. It is generally believed that the ball mill has good grinding efficiency when the interstitial filling fraction of the material is 0.6-1.2 [49, 50]. The parameter setting of the ball mill is shown in Table 1.

2.5. Process Parameters and Selection Basis. The process parameters were investigated based on patent CN104889411A [29]. The parameter combinations are shown in Table 2.

The densities of aluminum and graphite are similar. To ensure full contact between aluminum and graphite, the content of aluminum should not be less than that of graphite. On this basis, processes G-1 through G-4 with different combinations of aluminum particle size, aluminum content, and grinding time were obtained. It was found that graphene was obtained in G-1 and G-2 but was not obtained in G-3 or G-4. This may be because the energy of the steel balls could not be effectively transferred to the material when the particle size of aluminum powder was too small. 304 stainless-steel grit with a particle size of $0.4 \mathrm{~mm}$ was added to effectively disperse energy and increase the number of contact points for load transfer. Thus, process combinations G-5 through G-8 were obtained. The volume of steel grinding grit was determined based on the volumes of graphite and 
TABLE 2: Process parameters and test results.

\begin{tabular}{lcccc}
\hline Process & Graphite/g & $\begin{array}{c}\text { Pluminum } \\
\text { powder/g }\end{array}$ & Steel grit/g & $\begin{array}{c}\text { Grinding } \\
\text { time/min }\end{array}$ \\
\hline G-1 & 1 & $3(1.2 \mathrm{~mm})$ & - & 90 \\
G-2 & 1 & $5(1.2 \mathrm{~mm})$ & - & 30 \\
G-3 & 2 & $4(40 \mathrm{mesh})$ & - & 30 \\
G-4 & 2 & $4(80 \mathrm{mesh})$ & - & 30 \\
G-5 & 2 & $4(80 \mathrm{mesh})$ & $9(0.4 \mathrm{~mm})$ & 30 \\
G-6 & 2 & $2(80 \mathrm{mesh})$ & $6(0.4 \mathrm{~mm})$ & 30 \\
G-7 & 2 & $4(40 \mathrm{mesh})$ & $9(0.4 \mathrm{~mm})$ & 30 \\
G-8 & 2 & $2(40 \mathrm{mesh})$ & $6(0.4 \mathrm{~mm})$ & 30 \\
\hline
\end{tabular}

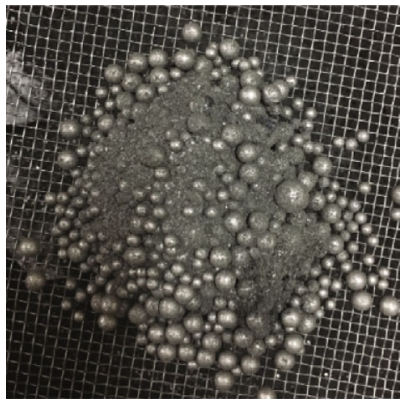

(a)

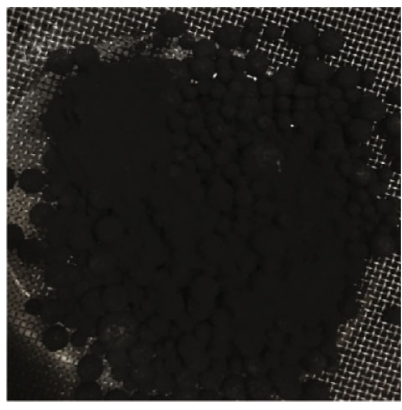

(b)
Figure 1: Two types of macroscopic appearance. (a) Silver-gray powder. (b) Pure black powder.

aluminum powder. The volume of the steel grit was chosen to be equal to that of the material to ensure effective contact and was then converted to the corresponding mass.

2.6. Microscopic Analysis of Graphene. The ground products showed two types of macroscopic appearance. As shown in Figure 1, one was silver-gray powder with metallic luster, and the other was pure black powder. According to the optical properties of graphene, the black powder can be used as an indicator to preliminary determine whether graphene is successfully prepared.

When light is shone on graphene, the electrons in the valence band (the energy band occupied by valence electrons) are excited to the conduction band (the energy space formed by free electrons) by absorbing the energy of the photons. The photoconductivity of single-layer graphene depends on the fine structure constant $\alpha$.

The linear transmittance of suspended single-layer graphene is[51]:

$$
T=\frac{1}{(1+0.5 \pi \alpha)^{2}} \approx 1-\pi \alpha \approx 97.7 \%
$$

where $\alpha$ is the fine structure constant, which is about $1 / 137$.

The light absorbance of single-layer graphene is $2.3 \%$. In the visible light region, the reflectance of single-layer graphene

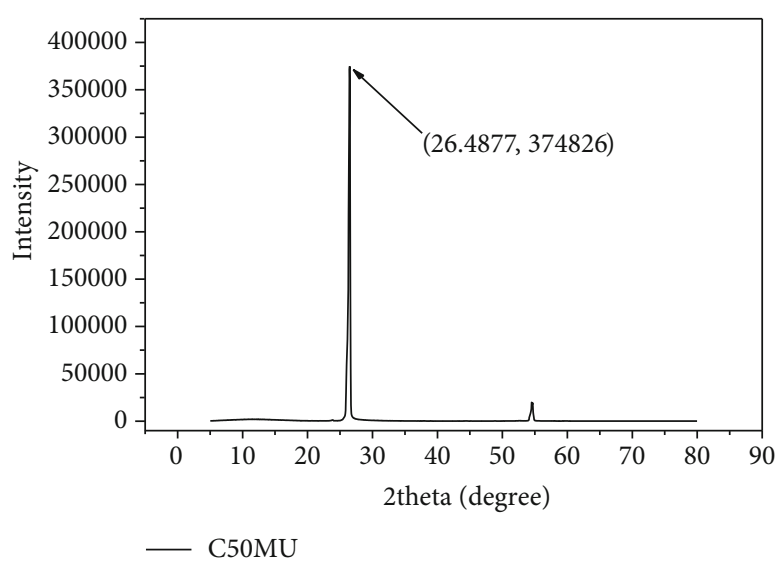

FIGURE 2: XRD pattern of flake graphite.

is less than $0.1 \%$, and the reflectance of 10-layer graphene is only $2 \%$, which suggests that graphene has extremely strong light absorption. The transmittance of single-layer graphene is as high as $97.7 \%$, and only $0.1 \%$ of the visible light will be reflected. Therefore, single-layer graphene is transparent under light. Within several layers of graphene, each additional layer increases the absorbance by $2.3 \%$. The few- and multiple-layer graphenes appear black due to their absorbance and reflectance [52]. Graphites with different structures have different physical properties, and the 50-mesh flake graphite itself has a silver-gray metallic luster.

The products of processes G-1 and G-2 were black powders (Figure 1(b)), which preliminarily indicated that the products contained a large amount of graphene. The grinding time of G-1 was too long, and the efficiency of G-1 was low compared to that of G-2. The G-2 product was selected for the microscopic characterization and was compared with DY-1 (graphene produced by Deyang Carbonene Technology Co., Ltd.). X-ray diffraction (XRD), specific surface area measurement, and HRTEM were performed for the microscopic characterization.

2.7. XRD Experiment. The principle of $\mathrm{X}$-ray crystal structure analysis is to measure the angle $\theta$ using the diffraction of $\mathrm{X}$ rays with a known wavelength and then calculate the crystal plane spacing $d$. X-rays are electromagnetic waves with short wavelengths (approximately 20 to $0.06 \AA$ ). They can generate diffraction peaks when passing through the gaps between the graphite layers. The interlayer spacing of graphite is $3.35 \AA$, and the corresponding peak of the $3.35 \AA$ crystal plane spacing is approximately $26^{\circ}$. The lower the peak intensity, the lower the crystal content and the higher the graphene content. XRD was performed with a D8 Advance diffractometer (Bruker, Germany). The result of the flake graphite analysis is shown in Figure 2.

Figure 2 shows that the peak of the graphite crystal appeared at approximately $26^{\circ}$. The peak value of the flake graphite crystal reached 374,826 , and the relative strength of the crystal was high, which indicated a good crystallinity of the sample. XRD was also performed on DY-1 and G-2 and is shown in Figure 3. 


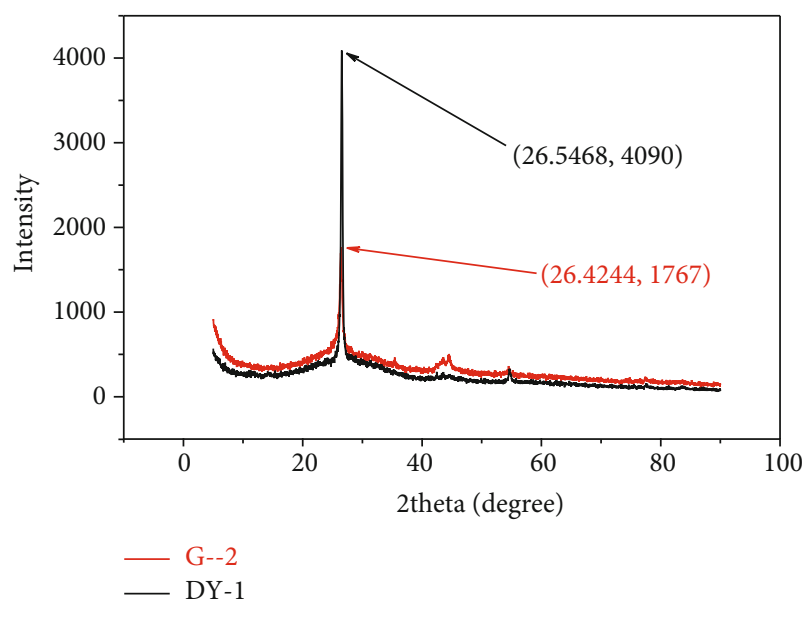

FIGURE 3: XRD patterns of DY-1 and G-2.

TABLE 3: BET test result.

\begin{tabular}{lc}
\hline Sample & Specific surface area $\left(\mathrm{m}^{2} / \mathrm{g}\right)$ \\
\hline DY-1 & 25.196 \\
G-2 & 502.177 \\
\hline
\end{tabular}

Figures 2 and 3 show that the peak value of DY-1 was 4090, which was higher than that of G-2 (1767). This result indicated that G-2 had lower graphite crystal content, higher graphene content, and therefore higher product quality than DY-1.

2.8. Brunauer-Emmett-Teller (BET) Experiment. The BET method for specific surface area measurement is widely used in the study of particle surface adsorption. It has become the most widely used and most accurate method in contemporary research [53]. The specific surface area measurement in this study was performed with a Quadrasorb SI instrument (Quantachrome Instruments). The result is shown in Table 3.

Table 3 shows that the specific surface area of G-2 was approximately 20 times that of DY-1. The adsorption capability of G-2 was much higher than that of DY-1.

\subsection{HRTEM Characterization of Graphene. A HRTEM is a} high-resolution transmission electron microscope that consists of an electro-optical part, a vacuum part, and an electronic part. An accelerated and focused electron beam is projected onto a target sample, and the electrons collide with the atoms in the sample and scatter. The scattering angle of the electron beam is related to information such as the density, thickness, and spacing of the sample. Thus, the electrons after the collision carry the relevant information of the sample, and such information can be converted back to images [54]. In this study, HRTEM was performed on a Tecnai G2 F20 field-emission TEM (FEI, USA). The characterization results of the two samples are shown in Figures 4 and 5.

Figures 4 and 5 show obvious layered structures in the two samples. G-2 and DY-1 show shadows in the $50 \mathrm{~nm}$ scanning range. Scanning the shadow in the $5 \mathrm{~nm}$ range, a large number of layers are observed. This is because the gra- phene obtained is not completely flat, and some of it has wrinkles due to curling. It can be seen that G-2 and DY-1 are not all single-layer graphene. It contains a small amount of single-layer graphene, but multilayer graphene [55] is the majority, so the sample of G-2 is not uniform. G-2 contained more dark spots in the $50 \mathrm{~nm}$ scanning range, which indicated that the impurity content in G-2 was higher than that in DY-1. Further cleaning and purification should be performed on G-2. In the application of engineering materials, graphene-modified asphalt has a very small amount of graphene. The effect of trace impurities in G-2 on engineering materials has yet to be verified.

Microscopic analysis and comparison of the two samples showed that the folds of sample DY-1 are more dense, which indicates that the G-2 prepared by the vibrating ball mill was significantly better than the DY-1 purchased on the market. However, the quality of the G-2 graphene was still not high. Therefore, further optimization of the process was needed.

\section{Process Optimization Design for Vibration Ball Milling}

3.1. Factor Selection. According to Table 2 and the preliminary test results, it was considered that the G-1, G-2, G-7, and G-8 processes were best out of the eight preparation processes. Therefore, XRD was performed on samples prepared by these four processes, and the results are shown in Figure 6.

It can be seen from Figure 6 that the peak intensity of G-1 was lower than that of G-2, but the efficiency of G-1 was low because the grinding time was too long. Therefore, the grinding time was fixed at $30 \mathrm{~min}$ to ensure grinding efficiency. G8 graphite had the lowest peak value, so the optimization was performed based on the G-8 process.

Through previous experience and exploration, we found that many factors affected the quality of the graphene, such as the particle size of the material, MBVR, grinding time, and the grading and content of the steel grit. Since there are many influencing factors and these factors interact with each other, some variables were determined first to reduce the number of experiments. The particle size of the aluminum powder was selected first by the control variates method based on the G-8 process. However, a large amount of chromium impurity was found in the G- 8 sample in the subsequent product purity test. It was determined that the chromium originated from the 40-mesh aluminum. Therefore, aluminum powders with four particle sizes (40 mesh, 100 mesh, 200 mesh, and 300 mesh) were reselected for testing. The process parameters are shown in Table 4.

Table 4 shows that the XRD peak intensity of G-9 multilayer graphene was higher than that of G-8 multilayer graphene, which indicates that chromium was helpful for the grinding of graphene. Although the G-8 multilayer graphene had higher quality, the chromium in the product could not be removed in the posttreatment, so the produced graphene could not be purified. In addition, the chromium content could not be quantitatively analyzed, and the calibration was difficult. Therefore, process G-8 was discarded. The experimental results of processes G-9 to G-12 were 


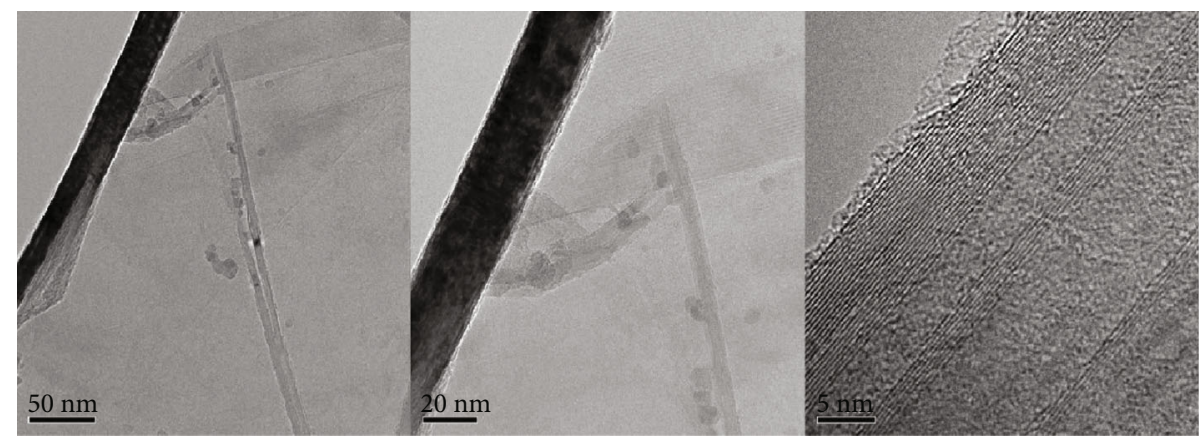

FIGURE 4: HRTEM of DY-1.

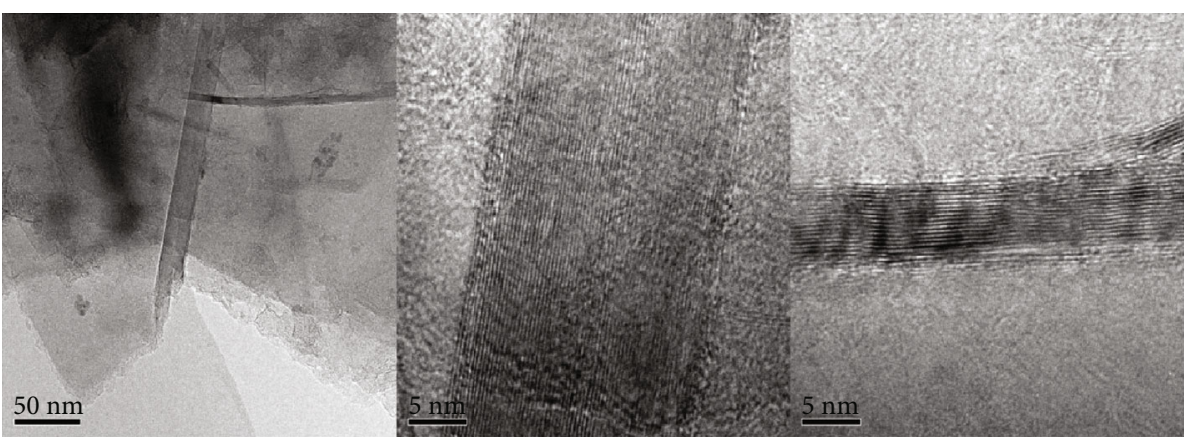

FIGURE 5: HRTEM of G-2.

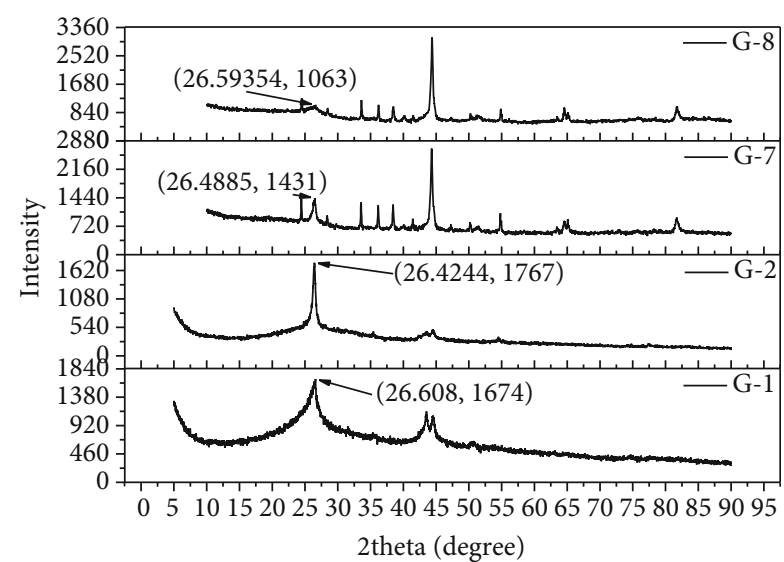

FIGURE 6: XRD results of samples prepared by the empirical process.

compared. The relationship between the peak value and the aluminum particle size is shown in Figure 7.

It can be seen from Figure 7 that under the same process conditions, the XRD peak intensity of the produced multilayer graphene was the lowest when the aluminum particle size was 200 mesh. Therefore, the aluminum particle size was first set at 200 mesh, and the design of process parameters was carried out based on this condition.

3.2. Process Parameter Design. Uniform experimental design was adopted for the process parameter design. The uniform experimental design was proposed by Chinese mathematicians Kaitai Fang and Yuan Wang in 1978. It is one of the main methods of fractional factorial design. Compared with the common orthogonal experimental design, the uniform experimental design gives the researchers more choices, and the desired results can be obtained with fewer experiments $[56,57]$. The percentage of voids $\mu$ between the grinding balls was measured to be 0.35 . Ball mills have a good grinding efficiency when the interstitial filling fraction of the material is 0.6-1.2 [51-53]. This study selected 1.2 as the limit value, and the amount of the material was controlled in this range. The limit amount of flake graphite (50 mesh) and aluminum (200 mesh) were both set to $5 \mathrm{~g}$ based on the size of the sample cylinder. Nine levels were fractioned in the range of $1 \mathrm{~g}$ to $5 \mathrm{~g}$. Three particle sizes $(0.2 \mathrm{~mm}, 0.4 \mathrm{~mm}$, and $0.8 \mathrm{~mm})$ of steel grit were selected. To construct a uniform design table with equal-level factors, the steel grit was set to have a single particle size or a mixture of multiple particle sizes. The three particle sizes were configured in accordance with the configuration principle of grinding balls. To facilitate the energy dispersion and increase the contact points, the following principles were employed: equal proportions, fewer $0.4 \mathrm{~mm}$ balls but more $0.2 \mathrm{~mm}$ and $0.8 \mathrm{~mm}$ balls, and fewer balls with larger sizes. The total mass of steel grit was kept unchanged at $6 \mathrm{~g}$ to ensure that the bulk volumes of steel grits were basically the same. In this paper, three factors were selected: $X_{1}$ - graphite content, $X_{2}$-aluminum content, and $X_{3}$-steel grit grading, each having 9 levels. The factor-level table is shown in Table 5.

The design was assisted by the Data Processing System (DPS) analysis software. DPS is a data processing system that integrates functions such as numerical calculation, statistical analysis, model simulation, and chart and table creation. It 
TABLe 4: Aluminum particle size selection scheme.

\begin{tabular}{|c|c|c|c|c|c|}
\hline \multirow{2}{*}{ Process } & \multicolumn{5}{|c|}{ Parameter } \\
\hline & Graphite/g & Aluminum/g & Steel grit/g & Grinding time/min & $\mathrm{XRD}$ result \\
\hline G-8 & 2 & 2 (40 mesh contains chromium) & $6(0.4 \mathrm{~mm})$ & 30 & 1063 \\
\hline G-9 & 2 & $2(40$ mesh $)$ & $6(0.4 \mathrm{~mm})$ & 30 & 2226 \\
\hline G-10 & 2 & $2(100$ mesh $)$ & $6(0.4 \mathrm{~mm})$ & 30 & 1452 \\
\hline G-11 & 2 & $2(200 \mathrm{mesh})$ & $6(0.4 \mathrm{~mm})$ & 30 & 1155 \\
\hline G-12 & 2 & 2 (300 mesh) & $6(0.4 \mathrm{~mm})$ & 30 & 1342 \\
\hline
\end{tabular}

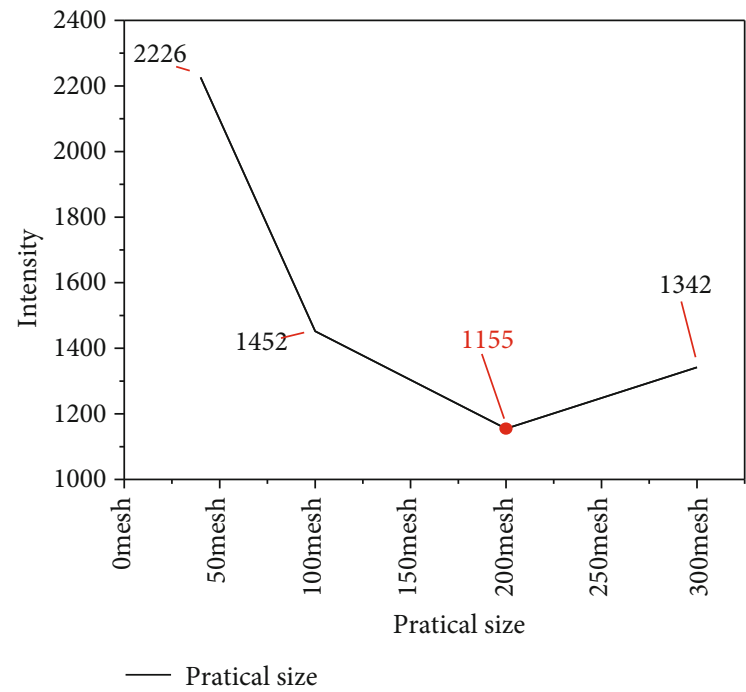

FIGURE 7: Relationship between XRD peak value and aluminum particle size.

has many experimental design functions, including uniform design and mixture uniform design $[58,59]$. The obtained uniform design scheme and the corresponding XRD results are shown in Table 6.

A mathematical model was established based on Table 6 with $X_{1}, X_{2}$, and $X_{3}$ as independent variables and $Y_{1}$ as a response variable. The independent variables $X_{1}$ and $X_{2}$ and the dependent variable $Y_{1}$ could be directly input into the model. However, the independent variable $X_{3}$ is a single-particle-size steel grit or a triple-particle-size steel grit (both types of grit had the same mass) and could not be directly inputted into the model. Therefore, the steel grit grading was first converted into a specific surface area and then inputted into the modeling calculation. The specific surface area of steel grit was calculated as follows:

$$
S=6 \div(\rho \times D)
$$

$\rho$-steel grit density- $7.93 \mathrm{~g} / \mathrm{cm}^{3}$

$D$-particle size

The specific surface areas of the steel grits with particle sizes of $0.8 \mathrm{~mm}, 0.4 \mathrm{~mm}$, and $0.2 \mathrm{~mm}$ calculated by equation (6) were $3 / 3172 \mathrm{~m}^{2} / \mathrm{g}, 3 / 1586 \mathrm{~m}^{2} / \mathrm{g}$, and $3 / 793 \mathrm{~m}^{2} / \mathrm{g}$, respectively. Then, the specific surface area corresponding to different $X_{3}$ can be obtained. At the same time, the G-13, G-14, and G-21 test groups with large $Y_{1}$ and obviously poor process capabilities were excluded from the test scheme. The results after data processing are shown in Table 7.

\section{Model Establishment and Solution}

A mathematical model was established using graphite concentration $\left(X_{1}\right)$, aluminum concentration $\left(X_{2}\right)$, and the specific surface area of steel grit $\left(X_{3}\right)$ as independent variables and the XRD peak intensity $\left(Y_{1}\right)$ as the response value. The model was optimized to obtain the preparation parameters. Graphene was prepared based on the simulated preparation parameters and was analyzed to verify the reliability and significance of the model.

4.1. Model Establishment. Partial least squares regression analysis is a method to study the quantitative relationship between multiple explanatory variables and multiple response variables. Therefore, the partial least squares quadratic polynomial regression, partial least squares (considering interaction terms) regression, and partial least squares (considering quadratic terms) regression were first selected in the DPS software to establish the model. The model calculation parameters are shown in Table 8.

Table 8 shows that when the model was established using the partial least squares (considering quadratic terms) regression, the sum of the squared errors was the smallest, the coefficient of determination $R^{2}$ was the highest (approaching 1), and the PRESS statistic was the smallest. Therefore, we chose to use the partial least squares (considering quadratic terms) regression for modeling.

First optimization (1stOpt) is a set of integrated software tools for mathematical analysis and optimization. It was independently developed by 7D-Soft High Technology Inc. (China) [60] and has strong computing ability in the fields of nonlinear regression, curve fitting, and parameter estimation for nonlinear complex engineering models. Therefore, the 1stOpt software was used for modeling, and the model was compared with that established by the DPS software. The partial least squares (considering quadratic terms) model was fitted by 1 stOpt $[61,62]$. The model parameters are shown in Table 9.

The closer the correlation coefficient is to 1 , the better the model is; the smaller the sum of squared residuals is, the better the model is. Therefore, the 1stOpt model was better than the DPS model. 
TABLE 5: Factor-level table for experimental design.

\begin{tabular}{|c|c|c|c|c|c|c|c|c|c|c|}
\hline \multirow{2}{*}{ Factor } & & \multicolumn{9}{|c|}{ Level } \\
\hline & & 1 & 2 & 3 & 4 & 5 & 6 & 7 & 8 & 9 \\
\hline$X_{1} / g$ & & 1 & 1.5 & 2 & 2.5 & 3 & 3.5 & 4 & 4.5 & 5 \\
\hline$X_{2} / g$ & & 1 & 1.5 & 2 & 2.5 & 3 & 3.5 & 4 & 4.5 & 5 \\
\hline \multirow{3}{*}{$X_{3} / g$} & $0.2 \mathrm{~mm}$ & 6 & 0 & 0 & 3 & 0 & 3 & 2 & 3 & 2.5 \\
\hline & $0.4 \mathrm{~mm}$ & 0 & 6 & 0 & 0 & 3 & 3 & 2 & 2 & 1 \\
\hline & $0.8 \mathrm{~mm}$ & 0 & 0 & 6 & 3 & 3 & 0 & 2 & 1 & 2.5 \\
\hline
\end{tabular}

TABLE 6: Uniform design scheme (note: the total mass of $X_{3}$ is kept at $6 \mathrm{~g} ; Y_{1}$ is the XRD result).

\begin{tabular}{lcccc}
\hline $\begin{array}{l}\text { Test } \\
\text { no }\end{array}$ & $\begin{array}{c}X_{1} \\
/ \mathrm{g}\end{array}$ & $\begin{array}{c}X_{2} \\
/ \mathrm{g}\end{array}$ & $0.2 \mathrm{~mm}: 0.8 \mathrm{~mm}=1: 1$ & $Y_{3}$ \\
\hline G-13 & 4 & 1 & 22461 \\
G-14 & 5 & 2.5 & $0.2 \mathrm{~mm}: 0.4 \mathrm{~mm}: 0.8 \mathrm{~mm}=1: 1: 1$ & 18493 \\
G-15 & 3 & 3 & $0.4 \mathrm{~mm}: 0.8 \mathrm{~mm}=1: 1$ & 4027 \\
G-16 & 2 & 5 & $0.2 \mathrm{~mm}: 0.4 \mathrm{~mm}=1: 1$ & 3794 \\
G-17 & 3.5 & 4 & $0.2 \mathrm{~mm}: 0.4 \mathrm{~mm}: 0.8 \mathrm{~mm}=2.5: 1: 2.5$ & 5132 \\
G-18 & 1.5 & 1.5 & $0.2 \mathrm{~mm}: 0.4 \mathrm{~mm}: 0.8 \mathrm{~mm}=3: 2: 1$ & 1403 \\
G-19 & 1 & 3.5 & $0.8 \mathrm{~mm}$ & 716 \\
G-20 & 2.5 & 2 & $0.2 \mathrm{~mm}$ & 2407 \\
G-21 & 4.5 & 4.5 & $0.4 \mathrm{~mm}$ & 20190 \\
\hline
\end{tabular}

TABLE 7: Summary of test results.

\begin{tabular}{lcccc}
\hline \multirow{2}{*}{ Test no. } & \multicolumn{4}{c}{ Factor } \\
& $X_{1} / \mathrm{g}$ & $X_{2} / \mathrm{g}$ & $X_{3} / \mathrm{m}^{2} / \mathrm{g}$ & $Y_{1}$ \\
\hline G-15 & 3 & 3 & 1.419 & 4027 \\
G-16 & 2 & 5 & 2.837 & 3794 \\
G-17 & 3.5 & 4 & 2.286 & 5132 \\
G-18 & 1.5 & 1.5 & 2.680 & 1403 \\
G-19 & 1 & 3.5 & 9.458 & 716 \\
G-20 & 2.5 & 2 & 3.783 & 2407 \\
\hline
\end{tabular}

The 1stOpt model is shown in equation (7):

$$
\begin{aligned}
& \begin{array}{l}
Y= \\
\quad \\
\quad \times X_{2}+84.2725+2363.46 \times X_{1}-1633.89417 \\
\quad \times X_{1}^{2}+318.2337448 \times X_{2}^{2}-87.32604572 \times X_{3}^{2}
\end{array} \\
& X_{1} \text { - graphite content }(\mathrm{g}) \\
& X_{2} \text { - aluminum content }(\mathrm{g}) \\
& X_{3} \text { - specific surface area of steel grit }\left(\mathrm{m}^{2} / \mathrm{g}\right) \\
& Y \text {-XRD peak value }
\end{aligned}
$$

4.2. Optimal Solution of the Model. The approach to obtain the optimal solution of the model is to find the values of $X_{1}$ , $X_{2}$, and $X_{3}$ so that $Y$ is equal to $0 . Y=0$ indicates that the crystal structure of graphite has completely disappeared, the graphene content in the product is high, the sample quality is high, and the purpose of optimization is achieved. Equa- tion (7) was solved by 1 stOpt taking into consideration the $\mathrm{XRD}$ results of the nine tests in the uniform design. When $X_{1}$ was greater than $X_{2}$, the XRD peak intensities of the obtained sample were all very high. To achieve a better exfoliation effect, the contents of graphite and aluminum should satisfy $X_{2} \geq X_{1}$, and the total mass of $X_{1}$ and $X_{2}$ must be kept within a reasonable range. If the volume is too large and the optimal range of the interstitial filling fraction (0.6-1.2) is exceeded, the grinding efficiency will decrease. Therefore, the constraint conditions were set as follows: $X_{1} \leq X_{2}$ and $X_{1}+X_{2} \leq 7 \mathrm{~g}$ (interstitial filling fraction $=0.6$ was set as the boundary condition). Since there are multiple solutions to the equation, the optimized solution summary is shown in Table 10 .

The $X_{3}$ obtained in Table 10 was the specific surface area, which had to be converted into a preparation parameter. The values of $X_{3}$ in Table 10 were compared with the values of $X_{3}$ in Table 7, but no corresponding values were found. Therefore, the particle size combination closest to each solution was first determined based on Table 7 . Two specific surface areas closest to each $X_{3}$ solution were chosen from Table 7 and were then converted to a combination of the steel grit particle sizes. The optimized experimental scheme is shown in Table 11.

4.3. Microscopic Analysis. Samples were prepared according to the six schemes in Table 10 and were characterized by XRD. The test results of samples prepared by the above six processes were compared with that of the best sample in Table 7 (G-19). The results are in Figure 8.

G-19 was the best process in the uniform design. G-22 to G-27 were processes obtained by model optimization. The XRD peak intensities of the G-22 to G-27 samples were lower than that of the G-19 sample, which indicated that the crystal contents of graphite in G-22 to G-27 were further reduced, and the qualities of multi-layer graphene in G-22 to G-27 products were improved. The effectiveness of process parameter optimization was confirmed. The peak value of the G-22 product was 134 , so G-22 was the best process in the current optimization scheme.

Representative processes (G-2, G-19, and G-22) were selected for specific surface area measurement. The BET results are shown in Table 12.

Table 12 shows that the specific surface area of the G-22 product was the largest. At the same time, the peak intensity of the G-22 product was the lowest by XRD. Therefore, the sample prepared by the G-22 process had the highest graphene content, and G-22 was the best process.

\section{Conclusion}

(1) Multilayer graphene with good quality can be effectively prepared by mechanical exfoliation of graphite by ball milling

(2) The process parameters of multilayer graphene production by a vibrating ball mill were designed and optimized using the uniform design method and mathematical modeling. The preparation materials 
TABLE 8: Standard sum of squared errors and coefficient of determination.

\begin{tabular}{lccc}
\hline \multirow{2}{*}{ Multiple regression method } & Sum of squared errors & Coefficient of determination $R^{2}$ & \multicolumn{2}{c}{ PRESS statistic } \\
& $Y_{1}$ & $Y_{1}$ & 4.6151 \\
\hline Partial least squares quadratic polynomial & 0.3066 & 0.9617 & 0.7824 \\
Partial least squares quadratic (considering interaction terms) & 1.7404 & 0.9775 & 5.7093 \\
Partial least squares quadratic (considering quadratic terms) & 0.1802 & 2.7284 \\
\hline
\end{tabular}

TABle 9: Model parameters.

\begin{tabular}{lcc}
\hline Model & Coefficient of determination $R^{2}$ & $\begin{array}{c}\text { Sum of squared } \\
\text { residuals (SSE) }\end{array}$ \\
\hline DPS model & 0.9775 & 0.1802 \\
1stOpt model & 1 & 0 \\
\hline
\end{tabular}

TABLE 10: Optimized solution.

\begin{tabular}{lcccc}
\hline Group & $X_{1} / \mathrm{g}$ & $X_{2} / \mathrm{g}$ & $X_{3} / \mathrm{m}^{2} / \mathrm{g}$ & $Y$ \\
\hline 1 & 1.0175 & 3.1831 & 3.1387 & 0 \\
2 & 0.7205 & 1.3377 & 2.4781 & 0 \\
3 & 1.0427 & 2.4808 & 2.9920 & 0 \\
\hline
\end{tabular}

TABle 11: Optimized experimental scheme.

\begin{tabular}{lccc}
\hline Test no. & $X_{1} / \mathrm{g}$ & $X_{2} / \mathrm{g}$ & $X_{3}$ \\
\hline G-22 & 1.0 & 3.2 & $0.2 \mathrm{~mm}$ \\
G-23 & 1.0 & 3.2 & $0.2 \mathrm{~mm}: 0.4 \mathrm{~mm}=1: 1$ \\
G-24 & 0.7 & 1.3 & $0.2 \mathrm{~mm}: 0.8 \mathrm{~mm}=1: 1$ \\
G-25 & 0.7 & 1.3 & $0.2 \mathrm{~mm}: 0.4 \mathrm{~mm}: 0.8 \mathrm{~mm}=3: 2: 1$ \\
G-26 & 1.0 & 2.4 & $0.2 \mathrm{~mm}$ \\
G-27 & 1.0 & 2.4 & $0.2 \mathrm{~mm}: 0.4 \mathrm{~mm}=1: 1$ \\
\hline
\end{tabular}

were flake graphite, 200-mesh aluminum powder, and 304 stainless-steel grit. The vibration frequency was $1200 \mathrm{r} / \mathrm{min}$, and the grinding time was $30 \mathrm{~min}$

(3) Many factors affect the multilayer graphene production by ball mills. In this study, the vibration frequency was $1200 \mathrm{r} / \mathrm{min}$, the static filling rate was $40 \%$, the MBVR was $51 \%$, and the void percentage of the media was 0.38 . More influencing factors can be selected in the future for further optimization of the preparation process

(4) Our findings show that for the preparation of multilayer graphene by a vibrating ball mill, the process parameters can be designed and optimized with the assistance of mathematic modeling. However, the yield of multilayer graphene produced in the laboratory was low

(5) This study provides a parameter design reference for the preparation of multilayer graphene by planetary ball mills

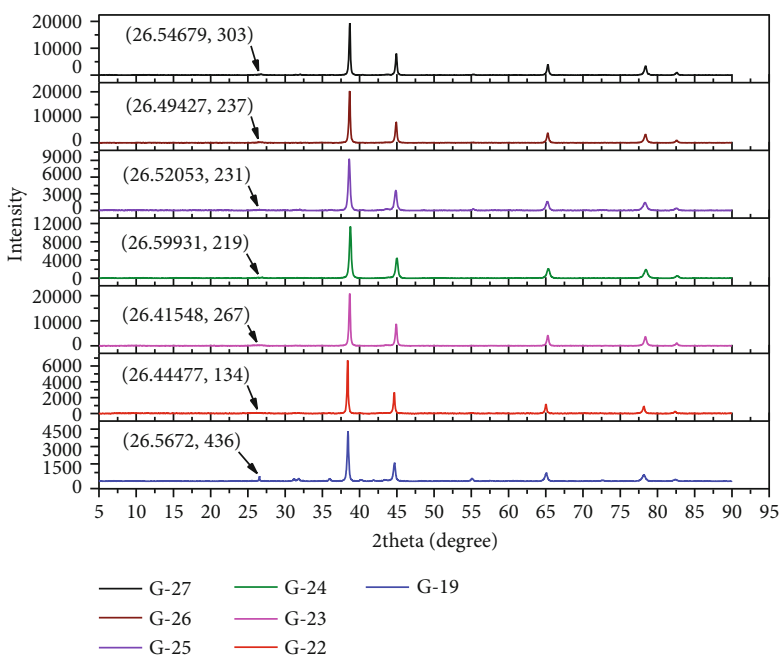

FIGURE 8: XRD results of samples prepared by seven processes (note: the background was subtracted from the G-19 XRD pattern in Figure 8).

TABle 12: Specific surface area results.

\begin{tabular}{lccc}
\hline Process & G-2 & G-19 & G-22 \\
\hline Specific surface area $/ \mathrm{m}^{2} / \mathrm{g}$ & 502.177 & 518.808 & 597.202 \\
\hline
\end{tabular}

\section{Data Availability}

The data used to support the findings of this study are included within the article.

\section{Conflicts of Interest}

The authors declare no conflict of interest.

\section{Acknowledgments}

The authors would like to gratefully acknowledge the financial supports from the National Natural Science Foundation of China and Municipal Foundation Project of CQEC and Natural Science Foundation Project of CQ CSTC. This research was funded by the National Natural Science Foundation of China (No.51778096) and the Natural Science Foundation Project of CQ CSTC (No. cstc2016jcyjA0119).

\section{References}

[1] Z. Chen and L. Gao, "Research progress on preparation and characterization of graphene," Development and Application of Materials, vol. 30, pp. 19-28, 2015. 
[2] K. S. Novoselov, A. K. Geim, S. V. Morozov et al., "Electric field effect in atomically thin carbon films," Science, vol. 306, no. 5696, pp. 666-669, 2004.

[3] A. K. Geim, "Graphene: status and prospects," Science, vol. 324, no. 5934, pp. 1530-1534, 2009.

[4] K. S. Novoselov, A. K. Geim, S. V. Morozov et al., "Twodimensional gas of massless dirac fermions in graphene," Nature, vol. 438, no. 7065, pp. 197-200, 2005.

[5] P. Solís-Fernández, M. Bissett, and H. Ago, "Synthesis, structure and applications of graphene-based 2D heterostructures," Chemical Society Reviews, vol. 46, no. 15, pp. 4572-4613, 2017.

[6] C. Lee, X. Wei, J. W. Kysar, and J. Hone, "Measurement of the elastic properties and intrinsic strength of monolayer graphene," Science, vol. 321, no. 5887, pp. 385-388, 2008.

[7] J. van den Brink, "From strength to strength," Nature Nanotechnology, vol. 2, no. 4, pp. 199-201, 2007.

[8] P. E. Steven, W. Swogger, D. P. Dubey, and N. Sugumaran, "Discrete carbon nanotubes increase lead acid battery charge acceptance and performance," Journal of Power Sources, vol. 261, pp. 55-63, 2014.

[9] A. S. Mayorov, R. V. Gorbachev, S. V. Morozov et al., "Micrometer-scale ballistic transport in encapsulated graphene at room temperature," Nano letters, vol. 11, no. 6, pp. 2396-2399, 2011.

[10] Z. Wang, Y. Qing, S. Meng, and Y. Shun, "Progress in preparation and application of graphene," Chemical Word, vol. 60, pp. 385-394, 2019.

[11] D. Li, M. B. Müller, S. Gilje, R. B. Kaner, and G. G. Wallace, "Processable aqueous dispersions of graphene nanosheets," Nature Nanotechnology, vol. 3, no. 2, pp. 101-105, 2008.

[12] W. Fu, Z. Zhang, P. Zhuang, J. Shen, and M. Ye, "One-pot hydrothermal synthesis of magnetically recoverable palladium/reduced graphene oxide nanocomposites and its catalytic applications in cross-coupling reactions," Journal of Colloid \& Interface Science, vol. 497, pp. 83-92, 2017.

[13] R. Kumar, R. K. Singh, A. R. Vaz, R. Savu, and S. A. Moshkalev, "Self-assembled and one-step synthesis of interconnected 3D network of Fe3O4/reduced graphene oxide nanosheets hybrid for high-performance supercapacitor electrode," ACS Applied Materials \& Interfaces, vol. 9, no. 10, pp. 8880-8890, 2017.

[14] A. V. Babichev, S. A. Rykov, M. Tchernycheva et al., "Influence of substrate microstructure on the transport properties of CVD-graphene," ACS Applied Materials \& Interfaces, vol. 8, no. 1, pp. 240-246, 2016.

[15] B. W. N. H. Hemasiri, J.-K. Kim, and J.-M. Lee, "Fabrication of highly conductive graphene/ITO transparent bi-film through CVD and organic additives-free sol-gel techniques," Scientific Reports, vol. 7, pp. 1786801-1786812, 2017.

[16] I. V. Komissarov, N. G. Kovalchuk, V. A. Labunov et al., "Nitrogen-doped twisted graphene grown on copper by atmospheric pressure CVD from a decane precursor," Beilstein Journal of Nanotechnology, vol. 8, pp. 145-158, 2017.

[17] N. Z. Habib, N. C. Aun, S. E. Zoorob, and P. I. Lee, "Use of graphene oxide as a bitumen modifier: an innovative process optimization study," Advanced Materials Research, vol. 1105, pp. 365-369, 2015.

[18] Y. Li, Preparation Technology of High Performance Graphene Modified Asphalt, [M.S. thesis], Chongqing Jiaotong University, 2018.
[19] J. X. He, Study on Material Composition Design Method and Mechanism of Graphene Modified Asphalt, [M.S thesis], Chongqing Jiaotong University, 2019.

[20] J. Zhang, Preparations, properties and applications of chemically reduced graphene oxide and its derivatives, [Ph.D. thesis], Shanghai Jiaotong University, 2011.

[21] S. Stankovich, D. A. Dikin, and R. D. Piner, "Synthesis of graphene-based nanosheets via chemical reduction of exfoliated graphite oxide," Carbon, vol. 45, no. 7, pp. 1558-1565, 2007.

[22] C. Nethravathi and M. Rajamathi, "Chemically modified graphene sheets produced by the solvothermal reduction of colloidal dispersions of graphite oxide," Carbon, vol. 46, no. 14, pp. 1994-1998, 2008.

[23] A. A. Balandin, S. Ghosh, W. Bao et al., "Superior thermal conductivity of single-layer graphene," Nano Letters, vol. 8, no. 3, pp. 902-907, 2008.

[24] L. S. Schadler, S. C. Giannaris, and P. M. Ajayan, "Load transfer in carbon nanotube epoxy composites," Applied Physics Letters, vol. 73, no. 26, pp. 3842-3844, 1998.

[25] V. D. B. Jeroen, "Graphene: from strength to strength," Australian Veterinary Journal, vol. 11, pp. 199-201, 2007.

[26] R. T. Weitz and A. Yacoby, "Nanomaterials: graphene rests easy," Nature Nanotechnology, vol. 5, no. 10, pp. 699-700, 2010.

[27] Q. Wang and J. Wang, "Influence of graphene oxide additions on the microstructure and mechanical strength of cement," New Carbon Materials, vol. 30, no. 4, pp. 349-356, 2015.

[28] J. Yu, Researeh \& development of vibration ball-milling machine for agricultural material processing, [M.S thesis], China Agricultural University, 2001.

[29] C. Zhou, L. Li, X. Yuan et al., 2015, Mechanical processing medium for preparing graphene, method for preparing graphene composite material and graphene, [p] CN104889411A.

[30] C. Zhou, L. Li, X. Yuan, L. He, and Z. He, 2016, Method for preparing graphene oxide, [p] CN103288078A.

[31] J. M. González-Domínguez, V. León, M. I. Lucío, M. Prato, and E. Vázquez, "Production of ready-to-use few-layer graphene in aqueous suspensions," Nature Protocols, vol. 13, no. 3, pp. 495-506, 2018.

[32] M. Buzaglo, I. P. Bar, M. Varenik, L. Shunak, S. Pevzner, and O. Regev, "Graphite-to-graphene: total conversion," Advanced Materials, vol. 29, no. 8, article 1603528, 2017.

[33] P. A. Cundall, "A computer model for simulating progressive large scale movements in blocky rock system," Proceedings of the Symposium of the International Society of Rock Mechanics, vol. 2, pp. 2-8, 1971.

[34] R. D. Morrison and P. W. Cleary, "Using DEM to model ore breakage within a pilot scale SAG mill," Minerals Engineering, vol. 17, no. 11-12, pp. 1117-1124, 2004.

[35] B. Liu, Research on Lifting Bar of Liner of Ball Mill Based on 3d Discrete Element Method, [M.S. thesis], Kunming University of Science and Technology, 2008.

[36] Y. Hou, Research on Performance of Large Tumbling Mill Liners Based on DEM-FEM Coupling Approach, [M.S. thesis], Jilin University, 2015.

[37] C. Huang, "Calculating means of the grinding mediums ratio of vibration mill," Guangdong Polytechnic of Water Resources and Electric Engineering, vol. 4, pp. 32-34, 2006.

[38] P. Li and M. Liu, "A research on theoretical rotative speed in ball mill and the optimum rotative speed in work," China Tungsten Industry, vol. 15, pp. 27-29,45, 2001. 
[39] Q. Tian, Study on Working Performances of Large Tumbling Ball Mill Based on Discrete Element Method, [M.S. thesis], Jilin University, 2011.

[40] Z. Wang, Study on measurement and optimization of the internal parameters in ball mill, [Ph.D. thesis], Northeastern University, 2001.

[41] Y. Hu and Q. Feng, Mineral Resources Processing Technology and Equipment, Science Press, 2006.

[42] L. Zhu, N. Zhang, and Y. Li, "Structure improvement of ball mill liner," Development Guide to Building Materials, vol. 5, pp. 51-53, 2007.

[43] E. Xia, W. Dong, and L. Ying, "Experimental research of the major factors influencing the medium movements in the ball mill," Mining Machinery Magazine, vol. 37, pp. 60-63, 2009.

[44] P. Huang, Study on Media Motion of Ball Mill Based on 3d Discrete Element Method, [M.S thesis], Kunming University of Science and Technology, 2007.

[45] H. Ren, Study on the Impact of Rotation Speed and Filling Rate to Grinding Effect, [M.S. thesis], Kunming University of Science and Technology, 2011.

[46] X. Duan, 2004, Ball milling machine for precise ball replacement, [p], no. CN1546237A.

[47] J. Sun, W. Dong, J. Zhang, and G. Shi, "Research on the influence of packing fraction and rotation rate on the power of ball mill," Mining Machinery Magazine, vol. 37, pp. 76-78, 2009.

[48] Z. Li, The study of flake graphite flotation characteristics, [Ph.D. thesis], China University Of Mining And Technology, Beijing, 2010.

[49] X. Duan and Y. Chao, Theory and Practice of Ball Mill Media Work, Metallurgical Industry Press, 2006.

[50] V. Deniz and T. Onur, "Investigation of the breakage kinetics of pumice samples as dependent on powder filling in a ball mill," International Journal of Mineral Processing, vol. 67, no. 1-4, pp. 71-78, 2002.

[51] R. R. Nair, P. Blake, A. N. Grigorenko et al., "Fine structure constant defines visual transparency of graphene," Science, vol. 320, no. 5881, pp. 1308-1308, 2008.

[52] W. Liao, Electronic and optical properties and its strain manipulation for graphene nanoribbons, [Ph.D. thesis], Hunan Normal University, 2010.

[53] X. Yang, Preparation of graphene-based composite materials and its adsorption properties for dyes, [Ph.D. thesis], Qingdao University, 2016.

[54] S. Wang, W. Jing, Z. Jiang, Q. Ling, F. Hang, and L. Li, “The measurement of single-layer thickness of graphene materials by high resolution transmission electron microscopy," Acta Metrologica Sinica, vol. 38, pp. 145-148, 2017.

[55] A. Bianco, H. M. Cheng, T. Enoki et al., "All in the graphene family - a recommended nomenclature for two-dimensional carbon materials," Carbon, vol. 65, pp. 1-6, 2013.

[56] K. Fang, "Uniform design-application of number theory method in experimental design," Acta Mathematicae Applicatae Sinica, vol. 3, pp. 363-372, 1980.

[57] Y. Wang and K. Fang, "About uniform distribution and experimental design (number theory method)," Science Bulletin, vol. 2, pp. 65-70, 1981.

[58] J. Wu, Z. Zhang, K. Chen, and L. Huang, "Application of case teaching method in biological statistics based on the DPS statistical software," Journal of Anhui Agricultural Sciences, vol. 41, no. 424, pp. 11234-11235, 2013.
[59] E. Zhang, Y. Zhao, and X. Song, "The application of DPS statistical software in the teaching of experiment design and statistical methods," Education Modernization, vol. 4, pp. 48-49, 2017.

[60] X. Ai, "Application of 1stopt in structural optimization design," Bullding Technique Development, vol. 34, p. 4-5+21, 2007.

[61] Z. Yan, "Research on gnss elevation fitting based on 1stopt software," East China University Of Technology, vol. 39, pp. 70-72, 2016.

[62] F. Wang, H. Wei, and Z. Li, "Viscoelastic parameter identification of asphalt mixture based on 1stopt method," Atlantis Press, vol. 22, pp. 2352-5401, 2018. 